\title{
Electronic Medical Records Optimize Clinical Data Management in the Outpatient Cardiological Clinic
}

\author{
S Dalmiani, MA Morales, C Carpeggiani, A Macerata*, P Marcheschi \\ CNR Clinical Physiology Institute, Pisa, Italy \\ * Department of Internal Medicine, Pisa University, Italy
}

\begin{abstract}
A system based on a relational database with administrative and clinical information and integrated with an Information System, where the system covers the role of a Functional Island, is routinely used in our Institution. To analyze how Electronic Medical Records (EMR) may help physicians in organizing and reducing time waste in a busy outpatient clinic, a sample of 1000 reports were evaluated for system performance. The time needed for building new clinical histories or modifying the already acquired ones, to create physical examination and biochemical and instrumental exams reports, was assessed. Cardio-pulmonary tests, basal, dynamic and effort EKG, chest X-ray, Echo Doppler studies, nuclear medicine procedures, invasive techniques, were collected.

Clinical management was integrated with the administrative system (ADT), to correctly identify each patient. This system provided not only easy retrieving of stored data such as clinical history, physical examination, instrumental exams reports but also the results of new exams performed in the same day of patient visit with a time lapse of 1 to 20'. Graphical interface structure contained preformatted fields which allowed selection or modifications of dataltext models without additional waste of time.

EMR represents an important tool for organization and synergy of different Laboratories in a outpatient clinic, optimizing the time needed for data entry and leading to an overall better quality of care.
\end{abstract}

\section{Introduction}

Improvement in diagnosis and treatment of cardiovascular diseases has led to overall prolongation of patient life, together with the need of periodical clinical assessments for further examinations, therapeutical changes and/or implementations.

The same patient can be admitted to the cardiological outpatient clinic many times/year for follow up. Most of the times, patients are provided with paper records of previous visits, instrumental examinations and biochemical analyses reports.

Clinical history compilation and physical examination reports can take a valuable amount of time. However, collection of paper reports of instrumental exams and their organization for correct interpretation and analysis, represent the most time consuming part of a clinical visit.

Aim of this study was to analyze how EMR may help physicians in organizing and reducing time waste through retrieving and collecting clinical and instrumental data available in the electronic archive implemented in our Institution.

Since bureaucratic procedures can also be time consuming, we analysed the interaction among clinical and administrative systems following the patient from his admission to the discharge.

\section{Materials \& methods}

Each week nearly 120 patients undergo a cardiological visit in our outpatient clinic. Each visit can be preceded by some instrumental examinations for a comprehensive assessment of patient current clinical status.

On a sample of 1000 consecutive visits we analyzed a typical clinical processing workflow, starting from administrative admission to the end of the visit.

To optimize such workflow, we developed a structured analysis of this process. The typical actions that a patient takes in the outpatient structure are shown in Figure 1.

The first step is represented by the administrative admission, which confirms patient arrival in the clinic followed by demographic recognition in the administrative electronic archive.

The informative systems which deal with administrative data, are frequently applied also in the clinical environment, due to their capability of economic and financial management for patient admission, discharge and transfer (ADT). A clinical informative system has to cope with ADT systems, reusing the collected data in order to avoid duplication of data entry (Figure 2). 


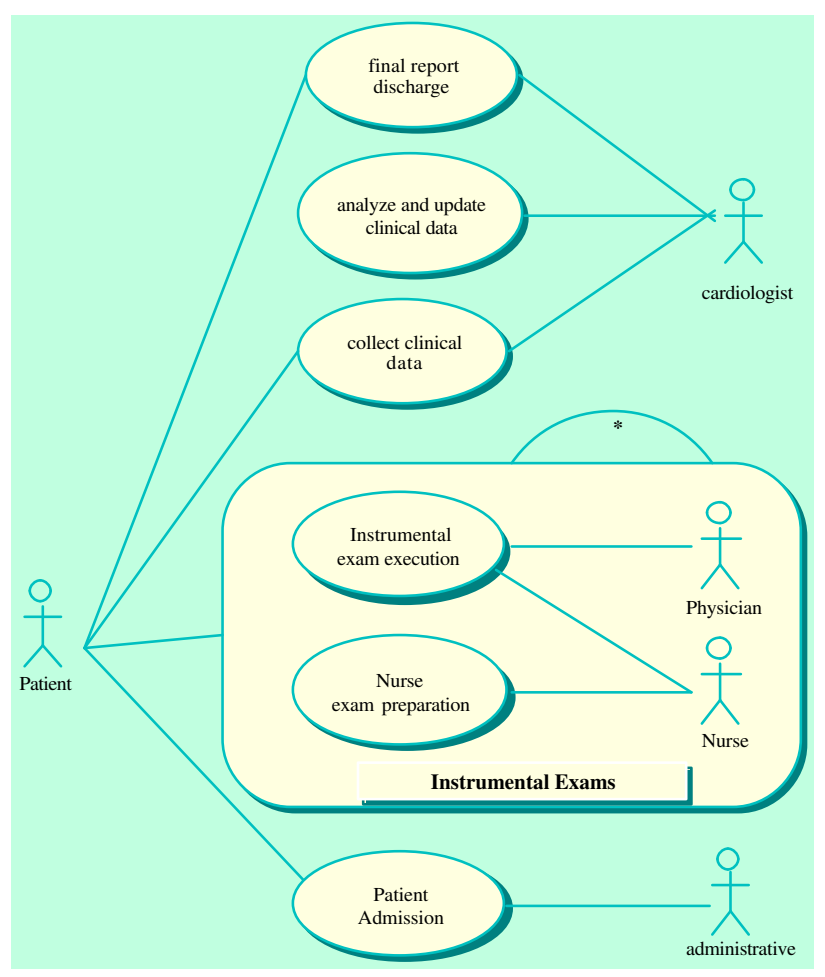

Figure 1 - Case analysis for a typical outpatient

A list of scheduled patients is built from the booking list and shown on the workstation placed in each visit and examination room. Therefore, as soon as the patient is available, and according to the order list, the programmed instrumental examinations and visits can be performed.

When all programmed laboratory exams are done, the patient can undergo the clinical visit. During this visit, an EMR system provides all previously acquired clinical and instrumental data which can be handled by the cardiologist (Figure 3).

\subsection{Methods}

To obtain a friendly and flexible system and to follow an evolving environment like cardiological outpatient care, a new development approach was adopted.

Extreme Programming (XP) [1] is a software development approach that represents an effective method for building smaller systems in an environment where requirements are changing continuously. XP methodology was originally applied to develop in-house information systems projects with a small developers team, like in our Institution [2].

$\mathrm{XP}$ is based on long standing industry best practices, including evolutionary prototyping, short release cycle and active end-user involvement in requirements definition [3]. XP contributes to bundle and package specific practices to form a methodology: planning game, pair programming, small releases, collective code ownership, metaphor, continuous integration, simple design, regression testing, on-site customer, continuous code refactoring, coding standard.

The core element of this system, partially inherited from the one developed for in-patients [4], is the clinical information system (SIC), which consists of many subsystems for laboratories and clinical environments.

The correct collection of patient demographics data allows a complete matching between the new patient and the existing ones together with individuation of already performed visits and exams.

In this process, clinical data are maintained on a separate archive from the administrative one in order to manage emergency situations such as ADT or network system failure.

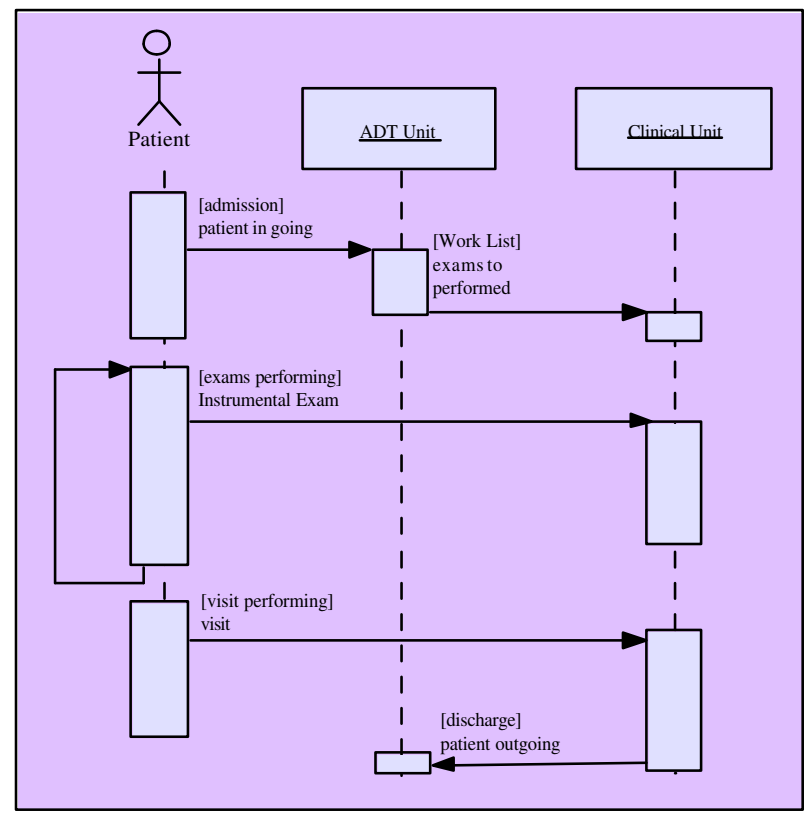

Figure 2 - Temporal administrative data flow

The clinical information system integrates different sources of information related to the single patient, represented by tests and examinations performed within specific diagnostic laboratories, which have been labelled "Functional Islands". The Functional Islands subsystems gather data from the internal archives to a central relational database, which duty is the integration of all information concerning the single patient to set up the EMR view.

Functional Islands consist of sub-units corresponding to peripheral collectors, providing local data management, numerical processing and digital archiving of images and biosignals as well as free texts and uploading to the central database of selected parts of the acquired data. 


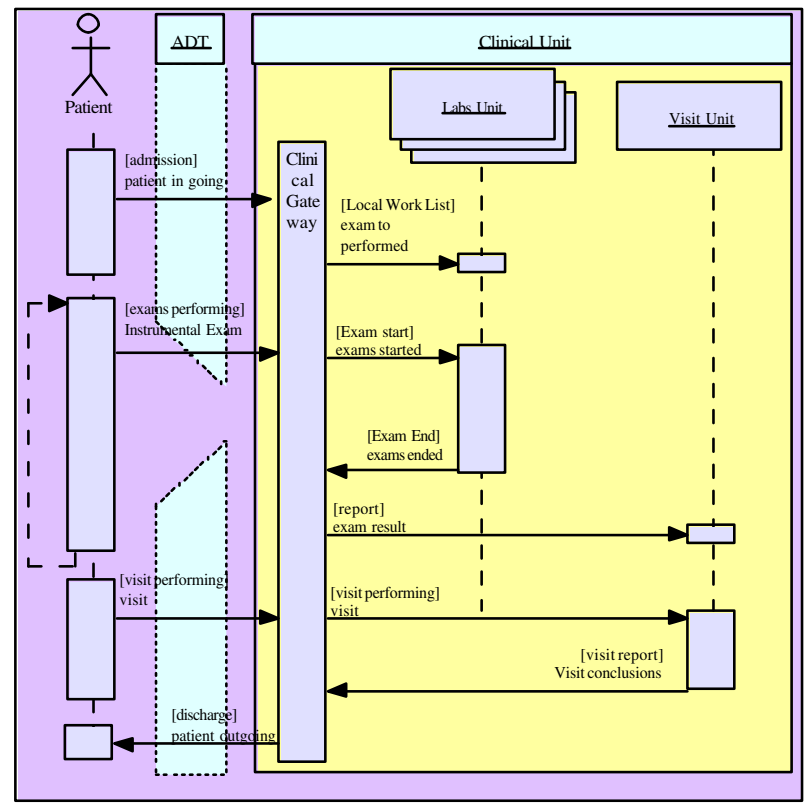

Figure 3 - Temporal clinical data flow: clinical unit details

One of the most common requirements for new software or systems is the capability to run on different machines and under different operating systems. In order to comply with these specifications, our EMR was developed in Java language [5], which guarantees a free integration on many popular operating systems and platforms and allows, through the JDBC protocol, an easy integration with many different database systems.

\section{Results}

The EMR working session is started by a passwordbased login procedure, which allows identification of the users and grant-related authorization. As soon as the patient undergoes the bureaucratic procedures, the Administrative system gives to the EMR the daily activity lists for each physician together with a time-track tool to manage work-lists and to handle situations like late arrival of the patient.

If the patient has been already evaluated in our Institute as out- or in- patient, the reports of the last clinical history becomes directly available in the EMR [6], ready for eventual modifications or adjuncts.

Without a previous history, an entire one should be typed. With this approach, history modifications do not take more than 1 min for update while a completely new one can take up to 8 ' to be collected and typed.

After that, physical examination (PE) is performed. The new EMR is provided with the reports of previous $\mathrm{PE}$, ready to be compared with current findings. On the same sample, this procedure needs less than 2' to be completed.

The evaluation of the reports coming from biochemical and instrumental Laboratories represents the most time consuming part of a cardiological visit.

This system is able to provide the results of exams performed even in the same day of patient visit (Figure 4), gathering reports coming from the different laboratories in 1 to 30 minutes after the instrumental exam is completed.

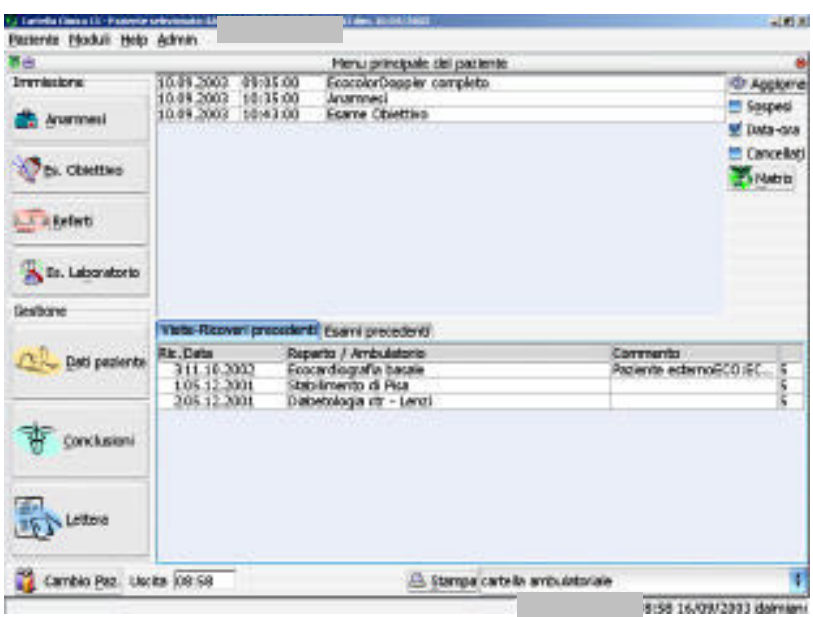

Figure 4 - EMR Java graphical interface

At the end of each visit, a printed report is given to the patient (Figure 5), containing data written by the specialist as clinical history, physical examination data, conclusions, therapy and follow up organization. and all the selected reports from different exams.

To allow remote and protected consultation, maintaining an open architecture, a WEB based report engine was developed to generate an HTML based printout, thus allowing also an external protected diffusion to other Specialists or family doctors provided with viewing permissions related to their patients.

This engine is used within a Servlet container [7] and manages the interaction with a WEB browser. Each document is based on an HTML model previously defined and stored in the database, for easy creation and update. The model definition contains position and format of displayed data, in a conventional and easy pattern, and is linked to the SQL query that collects desired data. 


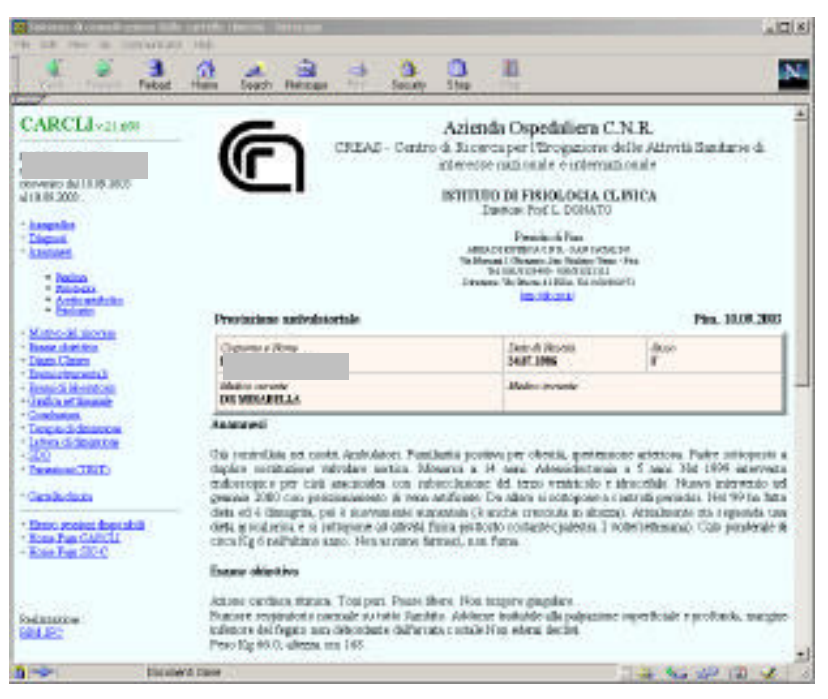

Figure 5 - Sample of visit discharge report on a Browser Window

\section{Conclusions}

In a busy cardiological outpatient clinic, organization, on both administrative and clinical side, is mandatory, in order to provide better performance avoiding unwanted waste of time.

The EMR developed in the Clinical Physiology Institute represents an important tool for the cardiologist for work organization even in the outpatient setting. It facilitates the interworking of multiple disciplines, starting from the administrative side. Confirmation of patient arrival at the administrative bureau allows to get not only retrievable data from previous exams, visits or hospitalization, but also tracking of the patient in the different outpatient laboratories, all provided with proper workstations.

Ease of use, retrieving of stored data, integration of instrumental examinations reports provided almost on line in the final report given to the patient, represent the most important aspects of our work. The presence of preformatted fields shortens the time needed to type clinical history to less than 8 ' also for patients undergoing a first visit, and to less than 1' for control visits as well as for updating physical examination reports.
In our opinion, the major advantage of the system is represented by the ease and promptness of obtaining results of the instrumental exams - from 1' to 20' after completion - even for those performed in the same day of the visit. This system property allows the performance of instrumental exams and clinical visit in the same day. This aspect is of great importance for all patients who live far from the Clinical Center and which can be followed with a smaller organizational effort.

This category of systems involves clinical personnel in a more accurate data entry and precision, different from generic knowledge of computerized systems. Although the theoretical learning curve could be more difficult to be followed, the final result in a trained medical and non medical personnel is a global gain in daily work.

Even if EMR use does not produce a direct lowering of patient care cost, in an environment with high rate of activity it represents the best available tool at present for work organization, leading to an overall better quality of care and structure efficiency.

\section{References}

[1] Beck K. Extreme Programming Explained: Embrace Change. Addison-Wesley, 1999; ISBN 201-61641-6.

[2] Macerata A, Landucci L, Pierotti D et al. Networking for health care administration and delivery: the information system at the CNR Institute of Clinical Physiology. In: Proceedings of Health Telematics '95.Pisa: 1995, 113-18.

[3] Fowler M, Planning Extreme Programming. AddisonWesley, 1999; ISBN 201-61641-6.

[4] Taddei A, Carpeggiani C, Emdin M et al: Development of an electronic medical record for patient care in Cardiology. In: Computers in Cardiology 1997; 22: 641-44.

[5] Sun Microsystems, Java 2 Platform, Standard Edition (J2SE) documentation. http://java.sun.com/docs/

[6] Morales MA, Dalmiani S, Carpeggiani C et al.: Electronic Medical Records in a Cardiological Outpatient Clinic In: Computers in Cardiology 2002; 29: 381-84.

[7] Apache Software Foundation, Tomcat documentation. http://jakarta.apache.org/tomcat/index.html

Address for correspondence.

Stefano Dalmiani.

CNR Institute of Clinical Physiology

Via Giuseppe Moruzzi 1

56124 Pisa, Italy

Phone: $\quad+390503152427$

Fax: $\quad+390503152311$

E-mail: dalmiani@ifc.cnr.it 